\title{
EXPLICIT EIGENVALUES AND INVERSES OF SEVERAL TOEPLITZ MATRICES
}

\author{
WEN-CHYUAN YUEH ${ }^{\otimes 1}$ and SUI SUN CHENG ${ }^{2}$
}

(Received 16 February, 2005; revised 3 March, 2006)

\begin{abstract}
Based on the theory of difference equations, we derive necessary and sufficient conditions for the existence of eigenvalues and inverses of Toeplitz matrices with five different diagonals. In the course of derivations, we are also able to derive computational formulas for the eigenvalues, eigenvectors and inverses of these matrices. A number of explicit formulas are computed for illustration and verification.

2000 Mathematics subject classification: primary 15A09, 15A18; secondary 39A10.

Keywords and phrases: Toeplitz matrix, eigenvalue, eigenvector, inverse, difference equation, sequence.
\end{abstract}

\section{Introduction}

A Toeplitz matrix is a matrix with values constant along each (top-left to lowerright) diagonal. Several properties of these matrices are now known, including their eigenvalues, eigenvectors and inverses. In particular, in a recent paper [4] by Dow, Toeplitz matrices of the form

$$
T_{n}=\left(\begin{array}{ccccccc}
b & c & 0 & 0 & \ldots & 0 & \alpha \\
a & b & c & 0 & \ldots & 0 & 0 \\
0 & a & b & c & \ldots & 0 & 0 \\
\ldots & \ldots & \ldots & \ldots & \ldots & \ldots & \ldots \\
\alpha & 0 & 0 & 0 & \cdots & a & b
\end{array}\right)_{n \times n},
$$

where the corner elements are the same, are discussed and their explicit inverses are found. In many applications (such as boundary value problems for difference

\footnotetext{
'Department of Refrigeration, Chin-Yi Institute of Technology, Taichung, Taiwan 411, R. O. China; e-mail: yuehwc@chinyi.ncit.edu.tw.

${ }^{2}$ Department of Mathematics, Tsing Hua University, Hsinchu, Taiwan 30043, R. O. China.

(C) Australian Mathematical Society 2006, Serial-fee code 1446-1811/06
} 
equations), Toeplitz matrices of the form $A_{n}=T_{n}$ but with the $\alpha$ in the bottom lefthand side comer replaced by $\beta$, that is, $\left[a_{n 1}\right]=\beta$, where $\alpha \neq \beta$, are also encountered. Therefore, it is of great interest to find out more about these matrices. In this paper, we derive the eigenvalues and their corresponding eigenvectors as well as the inverse of $A_{n}$ when $a c \neq 0$ and at least one of the numbers $\alpha$ or $\beta$ is not zero. When $\alpha=\beta=0, A_{n}$ reduces to the well-known tridiagonal matrix about which much is known. For general information about Toeplitz matrices, the references in [4] can be consulted.

For convenience, the set of integers, the set of nonnegative integers, the set of real numbers and the set of complex numbers are denoted by $\mathbb{Z}, \mathbb{N}, \mathbb{R}$ and $\mathbb{C}$ respectively. The number $\sqrt{-1}$ is denoted by $i$. We will also set

$$
\alpha \mathbb{Z}=\{m \alpha \mid m \in \mathbb{Z}\}, \quad \alpha \in \mathbb{C} .
$$

In particular, $\pi \mathbb{Z}$ denotes the set $\{\ldots,-2 \pi,-\pi, 0, \pi, 2 \pi, \ldots\}$.

Toeplitz matrices are intimately related to boundary value problems involving difference equations. This relationship has been exploited in $[2,10]$ for finding eigenvalues or inverses of matrices arising from difference operators. We will again base our investigation here on the method of difference equations. For this reason, we recall some terminologies used in [1]. Let $l^{\mathbb{N}}$ be the set of complex sequences of the form $x=\left\{x_{k}\right\}_{k \in \mathbb{N}}$ endowed with the usual linear structure. A sequence of the form $\{\alpha, 0,0, \ldots\}$ is denoted by $\bar{\alpha}$ (or by $\alpha$ if no confusion is caused), and the sequence $\{0,1,0,0, \ldots\}$ is denoted by $\hbar$. Given two sequences $x=\left\{x_{k}\right\}$ and $y=\left\{y_{k}\right\}$ in $l^{\mathbb{N}}$, their convolution is denoted by $x * y$ (or $x y$ if no confusion is caused) and is defined by

$$
x y=\left\{\sum_{k=0}^{j} x_{k} y_{j-k}\right\}_{j \in \mathbb{N}} .
$$

It is easily verified that $\hbar^{2}=\hbar * \hbar=\{0,0,1,0,0, \ldots\}$ and $\hbar^{n}=\left\{\hbar_{j}^{n}\right\}_{j \in \mathbb{N}}, n=$ $1,2, \ldots$, is given by $\hbar_{j}^{n}=1$ if $n=j$ and $\hbar_{j}^{n}=0$ otherwise. We will also set $\hbar^{0}=\overline{1}$.

In the following discussions, we need, among other things, the well-known properties of the complex functions $e^{z}, \sin z$ and $\cos z$ from the theory of complex analysis. In particular, let $z=x+i y \in \mathbb{C}$ where $x, y \in \mathbb{R}$. Then (i) $\sin z=0$ if and only if $y=0$ and $x=k \pi$ for some $k \in \mathbb{Z}$, (ii) $\cos z= \pm 1$ if and only if $y=0$ and $x=k \pi$ for some $k \in \mathbb{Z}$, and (iii) if $z \neq k \pi$ for any $k \in \mathbb{Z}$, then $\sin z \neq 0, \cos z \neq \pm 1$, $\sin (z / 2) \neq 0$ and $\cos (z / 2) \neq 0$.

\section{Necessary conditions for the eigenvalues}

Consider the eigenvalue problem $A_{n} u=\lambda u$, where $a, b, c$ and $\alpha, \beta$ are numbers in the complex plane $\mathbb{C}$. We will assume that $a c \neq 0$. When $a c=0$, the corresponding 
analysis is quite different and is treated elsewhere. To avoid trivial conditions, we will also assume $n \geq 3$ in the following discussions.

Let $\lambda$ be an eigenvalue (which may be complex) and $\left(u_{1}, \ldots, u_{n}\right)^{\dagger}$ a corresponding eigenvector of $A_{n}$. We may view the numbers $u_{1}, u_{2}, \ldots, u_{n}$ respectively as the first, second, $\ldots$, and the $n$-th term of an infinite (complex) sequence $u=\left\{u_{i}\right\}_{i=0}^{\infty}$. Since $A_{n} u=\lambda u$ can be written as

$$
\begin{aligned}
u_{0} & =0, \\
a u_{0}+b u_{1}+c u_{2} & =\lambda u_{1}-\alpha u_{n}, \\
a u_{1}+b u_{2}+c u_{3} & =\lambda u_{2}, \\
\ldots \ldots \ldots \cdots \cdots \cdots & \ldots \cdots \cdots \\
a u_{n-2}+b u_{n-1}+c u_{n} & =\lambda u_{n-1}, \\
a u_{n-1}+b u_{n}+c u_{n+1} & =\lambda u_{n}-\beta u_{1}, \\
u_{n+1} & =0,
\end{aligned}
$$

we see that the sequence $u=\left\{u_{k}\right\}_{k=0}^{\infty}$ satisfies $u_{0}=0, u_{n+1}=0$ and

$$
a u_{k-1}+b u_{k}+c u_{k+1}=\lambda u_{k}+f_{k}, \quad k=1,2, \ldots,
$$

where $f_{1}=-\alpha u_{n}$ and $f_{n}=-\beta u_{1}$, while $f_{k}=0$ for $k \neq 1, n$. Note that $u_{n}$ and $u_{1}$ cannot be 0 simultaneously, for otherwise from (2.1), $u_{2}=0$ and inductively $u_{3}=u_{4}=\cdots=u_{n}=0$, which is contrary to the definition of an eigenvector.

Let $f=\left\{f_{k}\right\}_{k=0}^{\infty}$ be defined above. Then (2.2) can be expressed as

$$
c\left\{u_{k+2}\right\}_{k=0}^{\infty}+b\left\{u_{k+1}\right\}_{k=0}^{\infty}+a\left\{u_{k}\right\}_{k=0}^{\infty}=\lambda\left\{u_{k+1}\right\}_{k=0}^{\infty}+\left\{f_{k+1}\right\}_{k=0}^{\infty} .
$$

By taking the convolution of the above equation with $\hbar^{2}=\hbar \hbar$, and noting that

$$
\hbar\left\{u_{n+1}\right\}=\hbar\left\{u_{1}, u_{2}, \ldots\right\}=\left\{0, u_{1}, u_{2}, \ldots\right\}=u-\bar{u}_{0}
$$

and

$$
\hbar^{2}\left\{u_{n+2}\right\}=\hbar^{2}\left\{u_{2}, u_{3}, \ldots\right\}=\left\{0,0, u_{2}, u_{3}, \ldots\right\}=u-\bar{u}_{0}-u_{1} \hbar,
$$

we have $c\left(u-\bar{u}_{0}-u_{1} \hbar\right)+(b-\lambda) \hbar\left(u-\bar{u}_{0}\right)+a \hbar^{2} u=\hbar\left(f-\bar{f}_{0}\right)$. Solving for $u$, and substituting $f_{0}=u_{0}=0$, we obtain

$$
\left(a \hbar^{2}+(b-\lambda) \hbar+\bar{c}\right) u=\left(c \bar{u}_{1}+f\right) \hbar .
$$

Since $c \neq 0$, we can divide the above equation by $a \hbar^{2}+(b-\lambda) \hbar+\bar{c}$ to obtain $[1$, Theorem 24]

$$
u=\frac{\left(c \bar{u}_{1}+f\right) \hbar}{a \hbar^{2}+(b-\lambda) \hbar+\bar{c}} .
$$


Let

$$
\gamma_{ \pm}=\frac{-(b-\lambda) \pm \sqrt{\omega}}{2 a}
$$

be the two roots of $a z^{2}+(b-\lambda) z+c=0$, where $\omega=(b-\lambda)^{2}-4 a c$, which may or may not be zero.

Based on the value of $\omega$, there are two cases to be considered.

Case I. Suppose $\omega \neq 0$ so that $\gamma_{+}$and $\gamma_{-}$are distinct. Since $\gamma_{+} \gamma_{-}=c / a \neq 0$, we may write $\gamma_{ \pm}=e^{ \pm i \theta} / \rho$ for some $\theta$ in the strip $\{z \in \mathbb{C} \mid 0 \leq \operatorname{Re} z<2 \pi\}$, where $\rho=\sqrt{a / c}$ and

$$
\cos \theta=(\lambda-b) / 2 \rho c .
$$

Since $\sin \theta=\sqrt{\omega} /(2 i \rho c) \neq 0$, we must also have $\theta \notin \pi \mathbb{Z}$.

By the method of partial fractions, we can then write (2.3) in the form

$$
\begin{aligned}
u & =\frac{1}{\sqrt{\omega}}\left(\frac{1}{\gamma_{-} \hbar}-\frac{1}{\gamma_{+}-\hbar}\right)\left(c \bar{u}_{1}+f\right) \hbar \\
& =\frac{1}{\sqrt{\omega}}\left\{\gamma_{-}^{-(j+1)}-\gamma_{+}^{-(j+1)}\right\}_{j \in \mathbb{N}}\left(c \bar{u}_{1}+f\right) \hbar \\
& =\frac{2 i}{\sqrt{\omega}}\left\{\rho^{j} \sin j \theta\right\} *\left\{c u_{1},-\alpha u_{n}, 0, \ldots,-\beta u_{1}, 0, \ldots\right\} .
\end{aligned}
$$

By evaluating the convolution product, we obtain the $j$-th term of $u$,

$$
\begin{aligned}
u_{j}= & \frac{2 i}{\sqrt{\omega}}\left(c u_{1} \rho^{j} \sin j \theta-\alpha u_{n} \rho^{j-1} \sin (j-1) \theta\right. \\
& \left.-H(j-n) \beta u_{1} \rho^{j-n} \sin (j-n) \theta\right)
\end{aligned}
$$

for $j \geq 1$, where $H(x)$ is the unit step function defined by $H(x)=1$ if $x \geq 0$ and $H(x)=0$ if $x<0$.

In particular,

$$
\frac{\sqrt{\omega}}{2 i} u_{n}=c u_{1} \rho^{n} \sin n \theta-\alpha u_{n} \rho^{n-1} \sin (n-1) \theta
$$

and

$$
\frac{\sqrt{\omega}}{2 i} u_{n+1}=c u_{1} \rho^{n+1} \sin (n+1) \theta-\alpha u_{n} \rho^{n} \sin n \theta-\beta u_{1} \rho \sin \theta .
$$

By (2.6), we have

$$
c \rho^{n}(\sin n \theta) u_{1}-\left(\frac{\sqrt{\omega}}{2 i}+\alpha \rho^{n-1} \sin (n-1) \theta\right) u_{n}=0
$$


and by (2.7), and the condition $u_{n+1}=0$, we have

$$
\left(c \rho^{n+1} \sin (n+1) \theta-\beta \rho \sin \theta\right) u_{1}-\alpha \rho^{n}(\sin n \theta) u_{n}=0 .
$$

Since $u_{1}$ and $u_{n}$ cannot be both zero, we must necessarily have

$$
\left|\begin{array}{cc}
c \rho^{n} \sin n \theta & -\left(\frac{\sqrt{\omega}}{2 i}+\alpha \rho^{n-1} \sin (n-1) \theta\right) \\
c \rho^{n+1} \sin (n+1) \theta-\beta \rho \sin \theta & -\alpha \rho^{n} \sin n \theta
\end{array}\right|=0,
$$

which leads to the necessary condition

$$
\alpha c \rho^{2 n} \sin \theta-\rho^{n}(a c \sin (n+1) \theta-\alpha \beta \sin (n-1) \theta)+a \beta \sin \theta=0 .
$$

Once we have found a $\theta$ that satisfies (2.10), we obtain by (2.4)

$$
\lambda=b+2 \rho c \cos \theta, \quad \theta \neq m \pi, m \in \mathbb{Z} .
$$

Case II. Suppose $\omega=0$ so that $\gamma_{+}=\gamma_{-}$. In this case, $(\lambda-b)^{2}=4 a c$, and (2.3) can be written as

$$
\begin{aligned}
u & =\frac{\left(c \bar{u}_{1}+f\right) \hbar}{c\left(\overline{1}-2((\lambda-b) / 2 c) \hbar+((\lambda-b) / 2 c)^{2} \hbar^{2}\right)}=\frac{1}{\widetilde{\rho} c} \frac{\tilde{\rho} \hbar}{(\overline{1}-\tilde{\rho} \hbar)^{2}}\left(c \bar{u}_{1}+f\right) \\
& =\frac{1}{\tilde{\rho} c}\left\{j \widetilde{\rho}^{j}\right\}_{j \in \mathbb{N}} *\left\{c u_{1},-\alpha u_{n}, 0, \ldots,-\beta u_{1}, 0, \ldots\right\},
\end{aligned}
$$

where

$$
\tilde{\rho}=(\lambda-b) / 2 c= \pm \sqrt{a / c}= \pm \rho .
$$

The $j$-th term of $u$ now becomes

$$
u_{j}=\frac{1}{\widetilde{\rho} c}\left(c u_{1} j \widetilde{\rho}^{j}-\alpha u_{n}(j-1) \tilde{\rho}^{j-1}-H(j-n) \beta u_{1}(j-n) \tilde{\rho}^{j-n}\right), \quad j \geq 1 .
$$

In particular,

$$
u_{n}=\left(c u_{1} n \widetilde{\rho}^{n}-\alpha u_{n}(n-1) \widetilde{\rho}^{n-1}\right) / \widetilde{\rho} c
$$

and

$$
u_{n+1}=0=\left(c u_{1}(n+1) \tilde{\rho}^{n+1}-\alpha u_{n} n \tilde{\rho}^{n}-\beta u_{1} \tilde{\rho}\right) / \tilde{\rho} c .
$$

This leads to the necessary condition

$$
\alpha c \widetilde{\rho}^{2 n}-\widetilde{\rho}^{n}(a c(n+1)-\alpha \beta(n-1))+a \beta=0 .
$$


Once we have found the $\widetilde{\rho}$ that satisfies (2.16), then we obtain by (2.12)

$$
\lambda=b+2 \widetilde{\rho} c .
$$

We remark that since (2.17) may be written as

$$
\lambda=b \pm 2 \rho c=b+2 \rho c \cos \theta, \quad \theta=m \pi, m \in \mathbb{Z},
$$

we may combine (2.17) and (2.11) and assert that an eigenvalue $\lambda$ of $A_{n}$ is necessarily of the form $\lambda=b+2 \rho c \cos \theta$.

According to the above discussions, when $\lambda$ is an eigenvalue of $A_{n}$, it is then necessary that either (2.10) or (2.16) holds.

THEOREM 2.1. Let $\lambda$ be an eigenvalue of the matrix $A_{n}$ and $u=\left(u_{1}, \ldots, u_{n}\right)^{\dagger}$ its corresponding eigenvector. If $(2.10)$ is satisfied for some

$$
\theta \in\{z \in \mathbb{C} \mid 0 \leq \operatorname{Re} z<2 \pi\} \backslash \pi \mathbb{Z}
$$

then (2.5) and (2.11) hold.

THEOREM 2.2. Let $\lambda$ be an eigenvalue of the matrix $A_{n}$ and $u=\left(u_{1}, \ldots, u_{n}\right)^{\dagger}$ its corresponding eigenvector. If (2.16) is satisfied for $\tilde{\rho}=\sqrt{a / c}$ or $\tilde{\rho}=-\sqrt{a / c}$, then (2.13) and (2.17) hold.

Recall that the first and last components $u_{1}$ and $u_{n}$ cannot be zero simultaneously. There are some other interesting properties for the eigenvector $u$ if $\omega \neq 0$.

COROLLARY 2.3. Let $\lambda$ be an eigenvalue of the matrix $A_{n}$ and $u=\left(u_{1}, \ldots, u_{n}\right)^{\dagger}$ its corresponding eigenvector such that $\omega=(b-\lambda)^{2}-4 a c \neq 0$. Let $\theta$ be the number found in Theorem 2.1.

(i) If $u_{1}=0$, then $\alpha \neq 0$.

(ii) If $u_{n}=0$ or $u_{1}=0$ then $\sin n \theta=0$.

(iii) If $\sin n \theta=0$, then either $u_{n}=0$ or $\alpha= \pm a \rho^{-n}$, and either $u_{1}=0$ or $\beta= \pm c \rho^{n}$.

(iv) If $\beta \neq \pm c \rho^{n}$, then $u_{n} \neq 0$.

(v) If $\alpha \neq \pm a \rho^{-n}$, then $u_{1} \neq 0$.

(vi) If $\beta \neq \pm c \rho^{n}$ and $u_{1} \neq 0$, then $\sin n \theta \neq 0$.

(vii) If $\alpha \neq \pm a \rho^{-n}$ and $u_{n} \neq 0$, then $\sin n \theta \neq 0$.

(viii) If $\beta \neq \pm c \rho^{n}$ and $\alpha \neq \pm a \rho^{-n}$, then $u_{1} \neq 0, u_{n} \neq 0$ and $\sin n \theta \neq 0$.

Proof. If $u_{1}=0$, then by $(2.8) \sqrt{\omega} /(2 i)+\alpha \rho^{n-1} \sin (n-1) \theta=0$. Since $\omega \neq 0$, we must have $\alpha \neq 0$. 
If $u_{n}=0$, then by (2.8) $c \rho^{n} u_{1} \sin n \theta=0$. Since $c \rho^{n} u_{1} \neq 0$, we must have $\sin n \theta=0$. Similarly, if $u_{1}=0$, then by (2.9) $\alpha \rho^{n} u_{n} \sin n \theta=0$. Since $\alpha \rho^{n} u_{n} \neq 0$, we must have $\sin n \theta=0$.

If $\sin n \theta=0$, then by (2.8), either $u_{n}=0$ or $\rho c \sin \theta+\alpha \rho^{n-1} \sin (n-1) \theta=0$. Since $\sin (n-1) \theta=\sin n \theta \cos \theta-\cos n \theta \sin \theta=\mp \sin \theta$ when $\sin n \theta=0$; the latter implies $\rho c= \pm \alpha \rho^{n-1}$ or $\alpha= \pm a \rho^{-n}$. Similarly, if $\sin n \theta=0$, then by (2.9), either $u_{1}=0$ or $c \rho^{n+1} \sin (n+1) \theta-\beta \rho \sin \theta=0$. The latter implies $\beta= \pm c \rho^{n}$.

Suppose $\beta \neq \pm c \rho^{n}$. If $u_{n}=0$, then $\sin n \theta=0$. Since $u_{1} \neq 0$, (iii) implies $\beta= \pm c \rho^{n}$, which is a contradiction.

Suppose $\alpha \neq \pm a \rho^{-n}$. If $u_{1}=0$, then $\sin n \theta=0$. Since $u_{n} \neq 0$, (iii) implies $\alpha= \pm a \rho^{-n}$, which is a contradiction.

Suppose $\beta \neq \pm c \rho^{n}$ and $u_{1} \neq 0$. If $\sin n \theta=0$, then by (iii), either $u_{1}=0$ or $\beta= \pm c \rho^{n}$. This is a contradiction.

Suppose $\alpha \neq \pm a \rho^{-n}$ and $u_{n} \neq 0$. If $\sin n \theta=0$, then by (iii), either $u_{n}=0$ or $\alpha= \pm a \rho^{-n}$. This is a contradiction.

The last assertion (viii) follows from (iv), (v) and (vi), (vii).

\section{Additional conditions for eigenvectors}

Given an eigenvalue $\lambda$ of the matrix $A_{n}$ and its corresponding eigenvector $u=$ $\left(u_{1}, \ldots, u_{n}\right)^{\dagger}$, suppose $\omega \neq 0$ and let $\theta$ be the number found in Theorem 2.1. For $1 \leq j \leq n$, we have by $(2.5)$

$$
u_{j}=\frac{1}{\rho c \sin \theta}\left(c u_{1} \rho^{j} \sin j \theta-\alpha u_{n} \rho^{j-1} \sin (j-1) \theta\right), \quad j=1, \ldots, n .
$$

In the case when $\sin n \theta \neq 0$, we may find $u$ which are simpler in form. Indeed, suppose $\sin n \theta \neq 0$, then from (2.8),

$$
u_{1}=\frac{\left(\rho c \sin \theta+\alpha \rho^{n-1} \sin (n-1) \theta\right)}{c \rho^{n} \sin n \theta} u_{n}
$$

and from (2.9),

$$
\alpha u_{n}=\frac{\left(c \rho^{n+1} \sin (n+1) \theta-\beta \rho \sin \theta\right)}{\rho^{n} \sin n \theta} u_{1} .
$$

Substituting (3.2) into (3.1), we have

$$
\begin{aligned}
u_{j} & =\frac{1}{\rho c \sin \theta}\left(\frac{\rho^{j} \sin j \theta}{\rho^{n} \sin n \theta}\left(\rho c \sin \theta+\alpha \rho^{n-1} \sin (n-1) \theta\right) u_{n}-\alpha u_{n} \rho^{j-1} \sin (j-1) \theta\right) \\
& =\frac{u_{n}}{\rho c \sin \theta \sin n \theta}\left(\rho c \rho^{j-n} \sin \theta \sin j \theta+\alpha \rho^{j-1} \sin (n-j) \theta \sin \theta\right)
\end{aligned}
$$


for $j=1,2, \ldots, n$. By letting $u_{n}=\rho^{n} \sin n \theta$, we obtain

$$
u_{j}=\rho^{j} \sin j \theta+\frac{\alpha}{a} \rho^{j+n} \sin (n-j) \theta, \quad j=1, \ldots, n,
$$

which defines an eigenvector corresponding to $\lambda$ (if $\theta$ is found). Similarly, substituting (3.3) into (3.1), we may obtain

$$
u_{j}=\rho^{j} \sin (n+1-j) \theta+\frac{\beta}{c} \rho^{j-n} \sin (j-1) \theta, \quad j=1, \ldots, n .
$$

Suppose $\omega=0$. Then we have by (2.13)

$$
u_{j}=\frac{1}{\widetilde{\rho} c}\left(c u_{1} j \widetilde{\rho}^{j}-\alpha u_{n}(j-1) \widetilde{\rho}^{j-1}\right), \quad j=1, \ldots, n .
$$

In view of (2.14) and (2.15), a similar argument leads to

$$
u_{j}=\tilde{\rho}^{j} j+\tilde{\rho}^{j+n} \frac{\alpha}{a}(n-j), \quad j=1, \ldots, n
$$

and

$$
u_{j}=\widetilde{\rho}^{j}(n+1-j)+\widetilde{\rho}^{j-n} \frac{\beta}{c}(j-1), \quad j=1, \ldots, n .
$$

\section{Eigenvalues and eigenvectors of special Toeplitz matrices}

Now we can apply the results of the previous sections to find the eigenvalues and the corresponding eigenvectors of several Toeplitz matrices of the form $A_{n}$. For motivation, consider the case where $\alpha=\beta=0$. Then (2.16) is reduced to

$$
\widetilde{\rho}^{n} a c(n+1)=0
$$

which is not possible so that, in view of Theorem 2.2, $\omega \neq 0$ and $\lambda$ cannot be of the form $b \pm 2 \rho c$. In view of Theorem 2.1, (2.10) must hold for some $\theta \notin \pi \mathbb{Z}$, or, $\sin (n+1) \theta=0$ for some $\theta \notin \pi \mathbb{Z}$. Consequently, $\theta=k \pi /(n+1)$ for some $k \in \mathbb{Z}$ and $\theta \notin \pi \mathbb{Z}$. An eigenvalue $\lambda_{k}$ is then necessarily of the form

$$
\lambda_{k}=b+2 \rho c \cos \frac{k \pi}{n+1}, \quad k=1, \ldots, n .
$$

A corresponding eigenvector, from (3.4), is given by

$$
u_{j}^{(k)}=\rho^{j} \sin \frac{j k \pi}{n+1}, \quad j=1, \ldots, n,
$$

which has also been obtained in [10] and elsewhere. Finally, by reversing the arguments that lead to Theorem 2.1, we see that each $\lambda_{k}$ is an eigenvalue of $A_{n}$ and the corresponding vector $u^{(k)}=\left(u_{1}^{(k)}, \ldots, u_{n}^{(k)}\right)^{\dagger}$ defined by (4.1) is a corresponding eigenvector.

By similar ideas, we may now derive the eigenvalues and the corresponding eigenvectors for matrices of the form $A_{n}$. 
4.1. The case where $a=c= \pm \alpha$ and $\beta=0$, or $\alpha=0$ and $a=c= \pm \beta \quad$ We will use $[x]$ to denote the integral part of $x \in \mathbb{R}$. Note that $[(k-1) / 2]+[(k+2) / 2]=k$ for any positive integer $k$.

THEOREM 4.1. Suppose $\alpha=a=c$ and $\beta=0$, or, $\alpha=0$ and $\beta=c=a$. Then the eigenvalues of $A_{n}$ are given by

$$
\lambda_{k}=b+2 a \cos \frac{2 k \pi}{n}, \quad k=1,2, \ldots,[(n-1) / 2]
$$

and

$$
\lambda_{m+[(n-1) / 2]}=b+2 a \cos \frac{(2 m-1) \pi}{n+2}, \quad m=1,2, \ldots,[(n+2) / 2] .
$$

The eigenvectors corresponding to (4.2) and (4.3) are given by

$$
u_{j}^{(k)}=\sin \frac{2 k(n+1-j) \pi}{n}, \quad j=1,2, \ldots, n
$$

and

$$
u_{j}^{(m+[(n-1) / 2])}=\sin \frac{(2 m-1)(n+1-j) \pi}{n+2}, \quad j=1,2, \ldots, n
$$

respectively for $\alpha=a=c$ and $\beta=0$, and

$$
u_{j}^{(k)}=\sin \frac{2 k j \pi}{n} \quad \text { and } \quad u_{j}^{(m+[(n-1) / 2])}=\sin \frac{(2 m-1) j \pi}{n+2}, \quad j=1,2, \ldots, n
$$

respectively for $\alpha=0$ and $\beta=c=a$.

PROOF. Suppose $\alpha=a=c$ and $\beta=0$, or $\alpha=0$ and $\beta=c=a$. Then (2.16) is reduced to $( \pm 1)^{n}(n+1)=1$. This relation cannot be valid, and hence, in view of Theorem 2.2, $\omega=0$ does not hold. In view of Theorem 2.1, (2.10) holds for some $\theta \notin \pi \mathbb{Z}$, or

$$
\sin \frac{n \theta}{2} \cos \left(\frac{n+2}{2}\right) \theta=0, \quad \theta \notin \pi \mathbb{Z} .
$$

Hence (a) $\sin (n \theta / 2)=0$ or (b) $\cos ((n+2) / 2) \theta=0$ for some $\theta \notin \pi \mathbb{Z}$. In case (a), we have

$$
\theta=2 k \pi / n, \quad \theta \notin \pi \mathbb{Z}, k \in \mathbb{Z},
$$

so that an eigenvalue must be of the form

$$
\lambda_{k}=b+2 a \cos (2 k \pi / n), \quad k=1, \ldots,[(n-1) / 2] .
$$

Similarly, in case (b), we have

$$
\theta=\frac{(2 m-1) \pi}{n+2}, \quad \theta \notin \pi \mathbb{Z}, m \in \mathbb{Z},
$$


so that an eigenvalue must be of the form

$$
\lambda_{m+[(n-1) / 2]}=b+2 a \cos \frac{(2 m-1) \pi}{n+2}, \quad m=1, \ldots,[(n+2) / 2] .
$$

The corresponding eigenvectors may be obtained as follows. For $\alpha=a=c$ and $\beta=0$, since $\beta \neq \pm c \rho^{n}$, by Corollary 2.3 (iv) $u_{n} \neq 0$, while $u_{1}$ may or may not be 0 . If $u_{1}=0$, then $\sin n \theta=0$. By (3.1)

$$
u_{j}=\frac{-u_{n}}{\sin \theta} \sin (j-1) \theta .
$$

Since $\sin (n+1-j) \theta=\sin n \theta \cos (j-1) \theta-\cos n \theta \sin (j-1) \theta=\mp \sin (j-1) \theta$ when $\sin n \theta=0$, we may write $u_{j}=\left( \pm u_{n} / \sin \theta\right) \sin (n+1-j) \theta$. Letting $\pm u_{n}=\sin \theta$, we have

$$
u_{j}=\sin (n+1-j) \theta, \quad j=1,2, \ldots, n .
$$

If $u_{1} \neq 0$, then by Corollary 2.3 (vi), $\sin n \theta \neq 0$. Hence we may apply (3.5), which leads to the same result (4.8) since $\rho=1$ and $\beta=0$. By substituting $\theta$ given by (4.6) or (4.7) into (4.8), we obtain the desired results (4.4) and (4.5).

For $\alpha=0$ and $\beta=c=a$, a similar argument leads to $u_{j}=\sin j \theta, j=1, \ldots, n$. By substituting $\theta$ given by (4.6) or (4.7), we obtain the desired results.

Once we have found the eigenvalues and their corresponding eigenvectors, we may reverse the arguments leading to Theorem 2.1 and verify that they are indeed the true eigenvalues and associated eigenvectors of $A_{n}$. The proof is complete.

We may follow the same arguments to show the following.

Suppose $-\alpha=a=c$ and $\beta=0$, or $\alpha=0$ and $-\beta=c=a$. Then the eigenvalues of $A_{n}$ are given by

$$
\lambda_{k}=b+2 a \cos \frac{2 k \pi}{n+2}, \quad k=1,2, \ldots,[(n+1) / 2]
$$

and

$$
\lambda_{m+[(n+1) / 2]}=b+2 a \cos \frac{(2 m-1) \pi}{n}, \quad m=1,2, \ldots,[n / 2] .
$$

The eigenvectors corresponding to (4.9) and (4.10) are given by

and

$$
u_{j}^{(k)}=\sin \frac{2 k(n+1-j) \pi}{n+2}, \quad j=1,2, \ldots, n
$$

$$
u_{j}^{(m+[(n+1) / 2])}=\sin \frac{(2 m-1)(n+1-j) \pi}{n}, \quad j=1,2, \ldots, n,
$$


respectively for $-\alpha=a=c$ and $\beta=0$, and

$$
u_{j}^{(k)}=\sin \frac{2 k j \pi}{n+2}, \quad j=1,2, \ldots, n
$$

and

$$
u_{j}^{(m+[(n+1) / 2])}=\sin \frac{(2 m-1) j \pi}{n}, \quad j=1,2, \ldots, n
$$

respectively for $\alpha=0$ and $-\beta=c=a$.

4.2. The case where $\alpha=-\beta=a=c$, or $-\alpha=\beta=a=c$

THEOREM 4.2. Suppose $\alpha=-\beta=a=c$, or $-\alpha=\beta=a=c$. Then the eigenvalues of $A_{n}$ are given by

$$
\lambda_{k}= \begin{cases}b+2 a \cos (k \pi / n), & k=1,2, \ldots, n-1, \\ b, & k=n .\end{cases}
$$

The eigenvectors corresponding to (4.11) are given by

$$
u_{j}^{(k)}= \begin{cases}\sin (j k \pi / n), & k \text { odd }, \\ \sin ((j-1) k \pi / n), & k \text { even }\end{cases}
$$

and

$$
u_{j}^{(n)}= \begin{cases}\sin (j \pi / 2)+(\alpha / a) \sin ((n-j) \pi / 2), & n \text { odd } \\ \sin (j \pi / 2), & n=6,10,14, \ldots \\ \sin ((j-1) \pi / 2), & n=4,8,12, \ldots\end{cases}
$$

respectively for $\alpha=-\beta=a=c, j=1,2, \ldots, n$. For $-\alpha=\beta=a=c$, only the odd-even relation for $k$ in (4.12) should be interchanged.

PROOF. Suppose $\alpha=-\beta=a=c$, or $-\alpha=\beta=a=c$, then $\rho=1$, and (2.16) is reduced to $( \pm 1)^{n} 2 n=0$. This relation cannot be valid so that $\omega=0$ does not hold. By Theorem 2.1, (2.10) holds for some $\theta \notin \pi \mathbb{Z}$, or $\sin n \theta \cos \theta=0, \theta \notin \pi \mathbb{Z}$. In the case where $\sin n \theta=0$ for some $\theta \notin \pi \mathbb{Z}$, we have $\theta=(k \pi / n) \notin \pi \mathbb{Z}, k \in \mathbb{Z}$, and the eigenvalue must be of the form $\lambda_{k}=b+2 a \cos (k \pi / n), k=1,2, \ldots, n-1$. In the case where $\cos \theta=0$, we have $\lambda_{n}=b$.

The corresponding eigenvectors may be found as follows. For $k=1, \ldots, n-1$, suppose $\alpha=-\beta=a=c$. Since $\sin n \theta=\sin k \pi=0$ and $\cos n \theta=\cos k \pi=-1$ if $k$ is odd and +1 if $k$ is even, we have

$$
\begin{array}{ll}
c \sin \theta+\alpha \sin (n-1) \theta=a \sin \theta(1-\cos n \theta)=2 a \sin \theta \neq 0, & k \text { odd, } \quad \text { and } \\
c \sin (n+1) \theta-\beta \sin \theta=c(\cos n \theta+1) \sin \theta=2 a \sin \theta \neq 0, & k \text { even. }
\end{array}
$$


Hence if $k$ is odd, by (2.8) $u_{n}=0$ and in view of (3.1) an eigenvector must be of the form

$$
u_{j}=\sin j \theta
$$

If $k$ is even, then by (2.9) $u_{1}=0$ and an eigenvector must be of the form

$$
u_{j}=\sin (j-1) \theta
$$

By substituting $\theta=k \pi / n$, we have (4.12). Suppose $-\alpha=\beta=a=c$, then (4.14) is for even $k$ and (4.15) is for odd $k$.

For $k=n$, we have $\theta=\pi / 2$ and $\sin n \theta=\sin (n \pi / 2)= \pm 1 \neq 0$ if $n$ is odd. We may apply (3.4) to obtain

$$
u_{j}^{(n)}=\sin \frac{j \pi}{2}+\frac{\alpha}{a} \sin \frac{(n-j) \pi}{2}, \quad j=1,2, \ldots, n .
$$

If $n$ is even, then $\sin n \theta=0$ and $\cos n \theta=-1$ if $n=6,10, \ldots$ and +1 if $n=4,8, \ldots$ Hence by a similar argument as for $k=1,2, \ldots, n-1$, we have for $j=1, \ldots, n$

$$
u_{j}^{(n)}= \begin{cases}\sin (j \pi / 2), & \text { for } n=6,10,14, \ldots \\ \sin ((j-1) \pi / 2), & \text { for } n=4,8,12, \ldots\end{cases}
$$

The proof is complete.

4.3. The case where $\alpha= \pm a$ and $\beta= \pm c \quad$ In the case where $\alpha=a$ and $\beta=c, A_{n}$ is the well-known circulant matrix [3]. There are many results [3,5-9] concerning the eigenvalues and inverses of such matrices. However, most of them are algorithmic in nature. Here we will derive explicit formulas for the case where $\alpha=-a$ and $\beta=-c$ based on our theorems, while those for $\alpha=a$ and $\beta=c$ will be listed only since they are already known.

THEOREM 4.3. Suppose $\alpha=-a$ and $\beta=-c$ in the matrix $A_{n}$. Then the eigenvalues and the corresponding eigenvectors of $A_{n}$ are given by

$$
\begin{aligned}
\lambda_{k}= & b+(a+c) \cos \frac{(2 k-1) \pi}{n} \\
& +i(a-c) \sin \frac{(2 k-1) \pi}{n}, \quad k=1,2, \ldots, n
\end{aligned}
$$

and

$$
u_{j}^{(k)}=e^{-i j(2 k-1) \pi / n}, \quad j=1,2, \ldots, n
$$


respectively. If $a=c$, an alternative formula for the eigenvectors is also given by

$$
u_{j}^{(k)}=c_{1} \cos \frac{(2 k-1) j \pi}{n}+c_{2} \sin \frac{(2 k-1) j \pi}{n}, j=1,2, \ldots, n,
$$

where $c_{1}$ and $c_{2}$ are two independent constants not both equal to 0 . In particular, if we take $c_{1}=1$ and $c_{2}=-i$, we have (4.17) as its special case.

PROOF. Suppose the conditions in Theorem 2.1 hold. Then we must have $\lambda=$ $b+2 \rho c \cos \theta$, where $\theta$ is some number that satisfies

$$
\rho^{n}(\sin (n+1) \theta-\sin (n-1) \theta)+\left(\rho^{2 n}+1\right) \sin \theta=0, \quad \theta \notin \pi \mathbb{Z},
$$

or, since $\sin \theta \neq 0, \rho^{2 n}+2 \rho^{n} \cos n \theta+1=0, \theta \notin \pi \mathbb{Z}$. This yields

$$
\rho^{n}=-\cos n \theta \pm i \sin n \theta=-e^{\mp i n \theta} .
$$

Let $\rho^{n}=-e^{i n \theta}$, then since $e^{ \pm i(2 k-1) \pi}=-1$, we may write $\rho^{n}=e^{i n \theta} e^{-i(2 k-1) \pi}$, so that

$$
e^{i \theta}=\rho e^{i(2 k-1) \pi / n}, \quad e^{-i \theta}=\rho^{-1} e^{-i(2 k-1) \pi / n}
$$

and

$$
\begin{aligned}
\cos \theta & =\frac{1}{2}\left(\rho e^{i(2 k-1) \pi / n}+\rho^{-1} e^{-i(2 k-1) \pi / n}\right) \\
& =\frac{1}{2}\left[\left(\rho+\frac{1}{\rho}\right) \cos \frac{(2 k-1) \pi}{n}+i\left(\rho-\frac{1}{\rho}\right) \sin \frac{(2 k-1) \pi}{n}\right] .
\end{aligned}
$$

Note that if $a \neq c$, then $\rho \neq 1$ and $\cos \theta \neq \pm 1$ for any $k \in \mathbb{Z}$. By noting that $\rho^{2} c=a$ and that $\sin x$ and $\cos x$ are periodic functions, we have finally for $k=1, \ldots, n$

$$
\lambda_{k}=b+2 \rho c \cos \theta=b+(a+c) \cos \frac{(2 k-1) \pi}{n}+i(a-c) \sin \frac{(2 k-1) \pi}{n},
$$

which is (4.16). If $a=c$, then $\rho=1$ and we have $\cos \theta=\cos ((2 k-1) \pi / n) \neq \pm 1$ so that $k \neq(n+1) / 2$ in (4.16). But then we have Theorem 2.2.

Suppose $\omega=0$ and the conditions in Theorem 2.2 hold. Then (2.16) is valid for $\alpha=-a$ and $\beta=-c$. Thus

$$
\widetilde{\rho}^{2 n}+2 \widetilde{\rho}^{n}+1=0,
$$

which holds if $a=c, \widetilde{\rho}=-1$ and $n$ is odd. Furthermore, under these conditions, the eigenvalue must be of the form $\lambda=b-2 c$, which can also be written as $\lambda_{(n+1) / 2}$ in (4.16). Hence (4.16) holds regardless of $a=c$ or $a \neq c$. 
In case the negative sign in $e^{\mp i n \theta}$ holds, then $\rho^{n}=-e^{-i n \theta}=e^{-i n \theta} e^{-i(2 k-1) \pi}$, and it is easily seen that we may get the same result (4.16).

To find the corresponding eigenvectors, we first consider the case $a \neq c$. Then $\rho \neq 1$ so that $\beta \neq \pm c \rho^{n}$ and $\alpha \neq \pm a \rho^{-n}$. By Corollary 2.3 (viii), $u_{1}, u_{2} \neq 0$ and $\sin n \theta \neq 0$, so we may apply (3.4). Since by (4.20) $e^{ \pm i j \theta}=\rho^{ \pm j} e^{ \pm i j(2 k-1) \pi / n}$, we have

$$
e^{ \pm i(n-j) \theta}=\rho^{ \pm(n-j)} e^{ \pm i(n-j)(2 k-1) \pi / n}=-\rho^{ \pm(n-j)} e^{\mp i j(2 k-1) \pi / n} .
$$

By substituting this and $\alpha=-a$ into (3.4),

$$
\begin{aligned}
u_{j}^{(k)}= & \frac{1}{2 i}\left(\rho^{j}\left(\rho^{j} e^{i j(2 k-1) \pi / n}-\rho^{-j} e^{-i j(2 k-1) \pi / n}\right)\right. \\
& \left.+\rho^{j+n}\left(\rho^{n-j} e^{-i j(2 k-1) \pi / n}-\rho^{-n+j} e^{i j(2 k-1) \pi / n}\right)\right) \\
= & \frac{1}{2 i}\left(\rho^{2 n}-1\right) e^{-i j(2 k-1) \pi / n} .
\end{aligned}
$$

By dropping the constant factor $\left(\rho^{2 n}-1\right) / 2 i$, we obtain (4.17). Next suppose $a=c$. By (4.19), $\rho=1$ implies $\cos n \theta=-1$ and $\sin n \theta=0$. The former implies $\theta=$ $(2 k-1) \pi / n \notin \pi \mathbb{Z}$ so that $k \neq(n+1) / 2$, the latter implies either one of the $u_{1}$ or $u_{n}$ may be zero. If $u_{n}=0$, then by (3.1) an eigenvector must be of the form $u_{j}^{(k)}=\sin j \theta$. If $u_{1}=0$, then an eigenvector must be of the form $u_{j}^{(k)}=\sin (j-1) \theta$. Hence the linear combination

$$
u_{j}^{(k)}=k_{1} \sin j \theta+k_{2} \sin (j-1) \theta=c_{1} \cos j \theta+c_{2} \sin j \theta
$$

is an eigenvector of $A_{n}$ corresponding to $\lambda_{k}$. After substituting $\theta=(2 k-1) \pi / n$, we have (4.18) for $a=c$ and $k \neq(n+1) / 2$.

For $a=c$ and $k=(n+1) / 2$, then $\theta=\pi$, which implies $\omega=0$, and we already have $\widetilde{\rho}^{n}=\widetilde{\rho}=-1$ from (4.21), hence we may apply either (3.6) or (3.7) to obtain

$$
u_{j}=(-1)^{j} n, \quad j=1, \ldots, n,
$$

which is of the form $u_{j}^{(n+1) / 2}$ in (4.18). Hence (4.18) is valid for $a=c$ and $k=1, \ldots, n$. The proof is complete.

Now we may follow the same arguments to show the following: Suppose $\alpha=a$ and $\beta=c$ in the matrix $A_{n}$, then the eigenvalues and the corresponding eigenvectors of $A_{n}$ are given by

$$
\lambda_{k}=b+(a+c) \cos \frac{2 k \pi}{n}+i(a-c) \sin \frac{2 k \pi}{n}, \quad k=1,2, \ldots, n
$$

and

$$
u_{j}^{(k)}=e^{-i \frac{2 j k \pi}{n}}, j=1,2, \ldots, n
$$


respectively. If $a=c$, an alternative formula for the eigenvectors is also given by [2]

$$
u_{j}^{(k)}=c_{1} \cos \frac{2 j k \pi}{n}+c_{2} \sin \frac{2 j k \pi}{n}, \quad j=1,2, \ldots, n,
$$

where $c_{1}$ and $c_{2}$ are two independent constants not both equal to 0 . In particular, if we take $c_{1}=1$ and $c_{2}=-i$, we have (4.22) as its special case.

\section{Necessary conditions for the inverse}

The method used in the previous sections may also be used to find the inverse of the matrix $A_{n}$ under the condition $a c \neq 0$. Let the (unique) inverse of $A_{n}$, if it exists, be denoted by

$$
G_{n}=\left(g^{(1)}\left|g^{(2)}\right| \cdots \mid g^{(n)}\right)=\left(\begin{array}{llll}
g_{1}^{(1)} & g_{1}^{(2)} & \ldots & g_{1}^{(n)} \\
g_{2}^{(1)} & g_{2}^{(2)} & \ldots & g_{2}^{(n)} \\
\cdots & \ldots & \ldots & \ldots \\
g_{n}^{(1)} & g_{n}^{(2)} & \ldots & g_{n}^{(n)}
\end{array}\right)_{n \times n}
$$

Then $A_{n} G_{n}=I_{n}$. We may view the numbers $g_{1}^{(k)}, g_{2}^{(k)}, \ldots, g_{n}^{(k)}$ respectively as the first, second, ..., and the $n$-th term of an infinite (complex) sequence $g^{(k)}=\left\{g_{j}^{(k)}\right\}_{j \in \mathrm{N}}$. Since $A_{n} G_{n}=I_{n}$ can be expanded as

$$
\begin{aligned}
& a g_{0}^{(k)}+b g_{1}^{(k)}+c g_{2}^{(k)}=\hbar_{1}^{k}-\alpha g_{n}^{(k)}, \\
& a g_{1}^{(k)}+b g_{2}^{(k)}+c g_{3}^{(k)}=\hbar_{2}^{k} \text {, } \\
& a g_{2}^{(k)}+b g_{3}^{(k)}+c g_{4}^{(k)}=\hbar_{3}^{k} \text {, } \\
& a g_{n-1}^{(k)}+b g_{n}^{(k)}+c g_{n+1}^{(k)}=\hbar_{n}^{k}-\beta g_{1}^{(k)} \text {, }
\end{aligned}
$$

with $g_{0}^{(k)}=g_{n+1}^{(k)}=0$, we have

$$
a g_{j-1}^{(k)}+b g_{j}^{(k)}+c g_{j+1}^{(k)}=\hbar_{j}^{k}+f_{j}^{(k)}, \quad j=1,2, \ldots
$$

where

$$
f_{j}^{(k)}= \begin{cases}-\alpha g_{n}^{(k)} & j=1 \\ -\beta g_{1}^{(k)} & j=n, \\ 0 & \text { otherwise }\end{cases}
$$

Since $c \neq 0$, we may obtain

$$
g^{(k)}=\frac{\left(c g_{1}^{(k)}+\hbar^{k}+f^{(k)}\right) \hbar}{a \hbar^{2}+b \hbar+\bar{c}}
$$


Let $\eta_{ \pm}=(-b \pm \sqrt{\xi}) / 2 a$ be the two roots of $a z^{2}+b z+c=0$, where $\xi=b^{2}-4 a c$. As in Section 2, there are two cases to be considered.

Case I. Suppose $\xi \neq 0$ so that $\eta_{+}$and $\eta_{-}$are two different numbers. Since $\eta_{+} \eta_{-}=$ $c / a \neq 0$, we may write $\eta_{ \pm}=e^{ \pm i \phi} / \rho$ for some $\phi$ in the strip $\{z \in \mathbb{C} \mid 0 \leq \operatorname{Re} z<2 \pi\}$, where $\rho=\sqrt{a / c}$ and

$$
\cos \phi=-b / 2 \rho c .
$$

We also have $\sin \phi=\sqrt{\xi} /(2 i \rho c) \neq 0$.

By the method of partial fractions, we may write $g^{(k)}$ in the form

$$
g^{(k)}=\frac{1}{\sqrt{\xi}}\left(\frac{1}{\eta_{-}-\hbar}-\frac{1}{\eta_{+}-\hbar}\right)\left(c g_{1}^{(k)}+\hbar^{k}+f^{(k)}\right) \hbar,
$$

which gives the $j$-th term of $g^{(k)}$ :

$$
\begin{aligned}
g_{j}^{(k)}= & \frac{2 i}{\sqrt{\xi}}\left\{c g_{1}^{(k)} \rho^{j} \sin j \phi-\alpha g_{n}^{(k)} \rho^{j-1} \sin (j-1) \phi\right. \\
& \left.+H(j-k) \rho^{j-k} \sin (j-k) \phi-H(j-n) \beta g_{1}^{(k)} \rho^{j-n} \sin (j-n) \phi\right\}
\end{aligned}
$$

for $j \geq 1$. In particular,

$$
\frac{\sqrt{\xi}}{2 i} g_{n}^{(k)}=c g_{1}^{(k)} \rho^{n} \sin n \phi-\alpha g_{n}^{(k)} \rho^{n-1} \sin (n-1) \phi+\rho^{n-k} \sin (n-k) \phi
$$

and

$$
\begin{aligned}
0= & c g_{1}^{(k)} \rho^{n+1} \sin (n+1) \phi-\alpha g_{n}^{(k)} \rho^{n} \sin n \phi \\
& -\beta g_{1}^{(k)} \rho \sin \phi+\rho^{n+1-k} \sin (n+1-k) \phi .
\end{aligned}
$$

If the inverse exists, then $g_{1}^{(k)}$ and $g_{n}^{(k)}$ form a unique solution pair and hence

$$
\Delta=\left|\begin{array}{cc}
c \rho^{n} \sin n \phi & -\left(\sqrt{\xi} / 2 i+\alpha \rho^{n-1} \sin (n-1) \phi\right) \\
c \rho^{n+1} \sin (n+1) \phi-\beta \rho \sin \phi & -\alpha \rho^{n} \sin n \phi
\end{array}\right| \neq 0,
$$

or

$$
\Delta=\left(\rho^{n}(a c \sin (n+1) \phi-\alpha \beta \sin (n-1) \phi)-\left(\alpha c \rho^{2 n}+a \beta\right) \sin \phi\right) \sin \phi \neq 0 .
$$

Furthermore, if $\Delta \neq 0$, then we have

$$
g_{1}^{(k)}=\Delta_{1} / \Delta, \quad g_{n}^{(k)}=\Delta_{n} / \Delta,
$$

where

$$
\begin{aligned}
& \Delta_{1}=-\alpha \rho^{2 n-k} \sin (k-1) \phi \sin \phi-a \rho^{n-k} \sin (n+1-k) \phi \sin \phi \quad \text { and } \\
& \Delta_{n}=-c \rho^{2 n+1-k} \sin k \phi \sin \phi-\beta \rho^{n+1-k} \sin (n-k) \phi \sin \phi .
\end{aligned}
$$


Case II. Suppose $\xi=0$ so that $\eta_{ \pm}$are two equal roots. In this case $b^{2}=4 a c$. Furthermore, from (5.2), we have

$$
\begin{aligned}
g^{(k)} & =\frac{\left(c g_{1}^{(k)}+\hbar^{k}+f^{(k)}\right) \hbar}{c\left(1-2(-b / 2 c) \hbar+(-b \hbar / 2 c)^{2}\right)} \\
& =\frac{1}{\rho c} \frac{\rho \hbar}{(1-\rho \hbar)^{2}}\left(c g_{1}^{(k)}+\hbar^{k}+f^{(k)}\right)=\frac{1}{\rho c}\left\{j \rho^{j}\right\} *\left(c g_{1}^{(k)}+\hbar^{k}+f^{(k)}\right),
\end{aligned}
$$

where $\rho=-b / 2 c$. The $j$-th term of $g^{(k)}$ is now

$$
\begin{aligned}
g_{j}^{(k)}= & \frac{1}{\rho c}\left\{c g_{1}^{(k)} j \rho^{j}-\alpha g_{n}^{(k)}(j-1) \rho^{j-1}\right. \\
& \left.+H(j-k)(j-k) \rho^{j-k}-H(j-n) \beta g_{1}^{(k)}(j-n) \rho^{j-n}\right\} .
\end{aligned}
$$

In particular, $g_{n}^{(k)}=\left(c g_{1}^{(k)} n \rho^{n}-\alpha g_{n}^{(k)}(n-1) \rho^{n-1}+(n-k) \rho^{n-k}\right) / \rho c$ and

$$
0=\frac{1}{\rho c}\left(c g_{1}^{(k)}(n+1) \rho^{n+1}-\alpha g_{n}^{(k)} n \rho^{n}+(n+1-k) \rho^{n+1-k}-\beta g_{1}^{(k)} \rho\right) .
$$

If the inverse exists, then $g_{1}^{(k)}$ and $g_{n}^{(k)}$ form a unique solution pair and hence we must have

$$
\Delta=-\alpha c \rho^{2 n}+\rho^{n}(a c(n+1)-\alpha \beta(n-1))-a \beta \neq 0,
$$

and

$$
g_{1}^{(k)}=\Delta_{1} / \Delta, \quad g_{n}^{(k)}=\Delta_{n} / \Delta
$$

where

$$
\begin{aligned}
& \Delta_{1}=-\rho^{2 n-k} \alpha(k-1)-\rho^{n-k} a(n+1-k) \quad \text { and } \\
& \Delta_{n}=-\rho^{2 n+1-k} c k-\rho^{n+1-k} \beta(n-k) .
\end{aligned}
$$

THEOREM 5.1. Let the inverse of the matrix $A_{n}$ be denoted by

$$
G_{n}=\left(g^{(1)}|\cdots| g^{(n)}\right) .
$$

If $b^{2}-4 a c \neq 0$, then the necessary and sufficient condition for the inverse to exist is that (5.5) holds for some $\phi \in\{z \in \mathbb{C} \mid 0 \leq \operatorname{Re} z<2 \pi\}$ that satisfies (5.3). Furthermore, if the inverse exists, then $g_{j}^{(k)}, 2 \leq j \leq n-1$, are given by (5.4), while $g_{1}^{(k)}$ and $g_{n}^{(k)}$ are given by (5.6). If $b^{2}-4 a c=0$, then the necessary and sufficient condition for the inverse to exist is that (5.10) holds. Furthermore, if the inverse exists, then $g_{j}^{(k)}, 2 \leq j \leq n-1$, are given by (5.9), while $g_{1}^{(k)}$ and $g_{n}^{(k)}$ are given by (5.11). 
We remark that sufficient conditions for the existence of the inverse of $A_{n}$ are added in the above result. This is valid since the above arguments can be reversed. We remark also that since $\cos z$ is $2 \pi$-periodic, the restriction $\phi \in\{z \in \mathbb{C} \mid 0 \leq \operatorname{Re} z<2 \pi\}$ can be relaxed to $\phi \in \mathbb{C}$. Furthermore, since if $b^{2} \neq 4 a c$, then $\cos \phi \neq \pm 1$ and $\phi \notin \pi \mathbb{Z}$ automatically (cf. Theorem 2.1).

\section{Inverses of some special Toeplitz matrices}

We may now apply Theorem 5.1 for finding the inverses for several special Toeplitz matrices. For motivation, consider the case where $\alpha=\beta=0$ in $A_{n}$. Let $g^{(k)}$ be the $k$-th column in the inverse $G_{n}$ of $A_{n}$. If $b^{2}=4 a c$, then by substituting $\alpha=\beta=0$ into (5.10) to (5.12), we have $\Delta=\rho^{n} a c(n+1) \neq 0$ and $\Delta_{1}=-\rho^{n-k} a(n+1-k)$. Substituting these into (5.9), we obtain

$$
\begin{aligned}
g_{j}^{(k)} & =\frac{1}{\rho c}\left(c g_{1}^{(k)} j \rho^{j}+H(j-k) \rho^{j-k}(j-k)\right) \\
& =\frac{-\rho^{j-k}}{\rho c(n+1)} \times \begin{cases}j(n+1-k), & j<k, \\
k(n+1-j), & j \geq k .\end{cases}
\end{aligned}
$$

After finding $g^{(k)}$, we may directly reverse the arguments leading to Theorem 5.1 and conclude that $G_{n}=\left(g^{(1)}\left|g^{(2)}\right| \cdots \mid g^{(n)}\right)$.

Now let us suppose that $b^{2} \neq 4 a c$. Suppose also that the inverse $G_{n}$ of $A_{n}$ exists and is of the form (5.1). Then substituting $\alpha=\beta=0$ into (5.5)-(5.7), in view of Theorem 5.1, we necessarily have $\cos \phi=-b / 2 \rho c, \phi \in \mathbb{C}$,

$$
\Delta=\rho^{n} a c \sin (n+1) \phi \sin \phi \neq 0 \quad \text { and } \quad \Delta_{1}=-a \rho^{n-k} \sin (n+1-k) \phi \sin \phi .
$$

Substituting these into (5.4), we have

$$
\begin{aligned}
g_{j}^{(k)} & =\frac{1}{\rho c \sin \phi}\left(\frac{-\rho^{j-k} \sin j \phi \sin (n+1-k) \phi}{\sin (n+1) \phi}+H(j-k) \rho^{j-k} \sin (j-k) \phi\right) \\
& =\frac{-\rho^{j-k}}{\rho c \sin \phi \sin (n+1) \phi} \times \begin{cases}\sin j \phi \sin (n+1-k) \phi, & j<k, \\
\sin k \phi \sin (n+1-j) \phi, & j \geq k .\end{cases}
\end{aligned}
$$

Once we have found $g_{j}^{(k)}$, then if $\sin (n+1) \phi \neq 0$, we may reverse the arguments leading to Theorem 5.1 and conclude that $\left(g^{(1)}\left|g^{(2)}\right| \cdots \mid g^{(n)}\right)$ is our desired inverse. On the other hand, if $\sin (n+1) \phi=0$, then $\Delta=0$ and by Theorem 5.1, the inverse of $A_{n}$ does not exist. 


\subsection{The case where $\alpha= \pm a$ and $\beta=0$}

THEOREM 6.1. Suppose $\alpha=a$ and $\beta=0$ in the matrix $A_{n}$.

(i) Suppose $b^{2} \neq 4 a c$. Then the inverse $G_{n}$ of $A_{n}$ given by (5.1) exists if, and only if, $\cos \phi=-b / 2 \rho c$ for some $\phi \in \mathbb{C}$ and $\sin (n+1) \phi-\rho^{n} \sin \phi \neq 0$. Furthermore, if it exists, then

$$
\begin{aligned}
g_{j}^{(k)}= & \frac{-\rho^{j-k}}{\rho c \sin \phi\left(\sin (n+1) \phi-\rho^{n} \sin \phi\right)} \\
& \times \begin{cases}\sin j \phi \sin (n+1-k) \phi+\rho^{n} \sin (k-j) \phi \sin \phi, & j<k, \\
\sin k \phi \sin (n+1-j) \phi, & j \geq k .\end{cases}
\end{aligned}
$$

(ii) Suppose $b^{2}=4 a c$. Then the inverse $G_{n}$ of $A_{n}$ given by (5.1) exists if, and only if, $n+1-\rho^{n} \neq 0$ and, if it exists,

$$
g_{j}^{(k)}=\frac{-\rho^{j-k}}{\rho c\left(n+1-\rho^{n}\right)} \times \begin{cases}j(n+1-k)+\rho^{n}(k-j), & j<k, \\ k(n+1-j), & j \geq k .\end{cases}
$$

PROOF. Suppose the inverse of $A_{n}$ exists and is of the form (5.1). If $b^{2} \neq 4 a c$, then substituting $\alpha=a$ and $\beta=0$ into (5.5)-(5.8), we necessarily have $\cos \phi=-b / 2 \rho c$, $\phi \in \mathbb{C}$,

$$
\begin{aligned}
\Delta & =a c \rho^{n}\left(\sin (n+1) \phi-\rho^{n} \sin \phi\right) \sin \phi \neq 0, \\
\Delta_{1} & =-a \rho^{n-k}\left(\rho^{n} \sin (k-1) \phi+\sin (n+1-k) \phi\right) \sin \phi \quad \text { and } \\
\Delta_{n} & =-c \rho^{2 n+1-k} \sin k \phi \sin \phi .
\end{aligned}
$$

Substituting these into (5.4), we obtain

$$
\begin{aligned}
g_{j}^{(k)}= & \frac{\rho^{j-k}}{\rho c \sin \phi}\left(\frac{\rho^{n} \sin (j-k) \phi \sin \phi-\sin (n+1-k) \phi \sin j \phi}{\sin (n+1) \phi-\rho^{n} \sin \phi}\right. \\
& +H(j-k) \sin (j-k) \phi)
\end{aligned}
$$

which is equivalent to (6.1).

Once we have found $g_{j}^{(k)}$, then if $\sin (n+1) \phi-\rho^{n} \sin \phi \neq 0$, we may verify that $G_{n}$ is the inverse of $A_{n}$. On the other hand, if $\sin (n+1) \phi-\rho^{n} \sin \phi=0$, then the inverse does not exist.

If $b^{2}=4 a c$, then by substituting $\alpha=a, \beta=0$ into (5.10) to (5.13), we necessarily have $\Delta=a c \rho^{n}\left(n+1-\rho^{n}\right) \neq 0, \Delta_{1}=-a \rho^{n-k}\left(\rho^{n}(k-1)+(n+1-k)\right)$ and $\Delta_{n}=-\rho^{2 n+1-k} c k$. Substituting these into (5.9), we obtain

$$
g_{j}^{(k)}=\frac{\rho^{j-k}}{\rho c}\left\{\frac{\rho^{n}(j-k)-j(n+1-k)}{n+1-\rho^{n}}+H(j-k)(j-k)\right\},
$$


which is equivalent to (6.2). Once we have found $g_{j}^{(k)}$, then if $n+1-\rho^{n} \neq 0$, we may verify that $G_{n}$ is the inverse of $A_{n}$. On the other hand, if $n+1-\rho^{n}=0$, then the inverse does not exist. The proof is complete.

Suppose $\alpha=-a$ and $\beta=0$ in the matrix $A_{n}$. We may follow the same arguments to show the following. (i) Suppose $b^{2} \neq 4 a c$, then the inverse $G_{n}$ of $A_{n}$ given by (5.1) exists if, and only if, $\cos \phi=-b / 2 \rho c$ for some $\phi \in \mathbb{C}$ and $\sin (n+1) \phi+\rho^{n} \sin \phi \neq 0$. Furthermore, if it exists, then

$$
\begin{aligned}
g_{j}^{(k)}= & \frac{-\rho^{j-k}}{\rho c \sin \phi\left(\sin (n+1) \phi+\rho^{n} \sin \phi\right)} \\
& \times \begin{cases}\sin j \phi \sin (n+1-k) \phi-\rho^{n} \sin (k-j) \phi \sin \phi, & j<k, \\
\sin k \phi \sin (n+1-j) \phi, & j \geq k .\end{cases}
\end{aligned}
$$

(ii) Suppose $b^{2}=4 a c$, then the inverse $G_{n}$ of $A_{n}$ given by (5.1) exists if, and only if, $n+1+\rho^{n} \neq 0$ and, if it exists,

$$
g_{j}^{(k)}=\frac{-\rho^{j-k}}{\rho c\left(n+1+\rho^{n}\right)} \times \begin{cases}j(n+1-k)-\rho^{n}(k-j), & j<k, \\ k(n+1-j), & j \geq k .\end{cases}
$$

As we have seen, the derivation of the explicit formulas from Theorem 5.1 are straightforward. Theoretically, we can obtain formulas for arbitrary $\alpha$ and $\beta$, though in most cases those formulas may be complicated in form. But at least we can obtain elegant formulas for some special combinations of $\alpha$ and $\beta$. Some of the results are presented below without proof for comparison and quick reference. The proof is simple and may be obtained in a way similar to that of Theorem 6.1.

\subsection{The case where $\alpha=0$ and $\beta= \pm c$}

THEOREM 6.2. Suppose $\alpha=0$ and $\beta=c$ in the matrix $A_{n}$.

(i) Suppose $b^{2} \neq 4 a c$. Then the inverse $G_{n}$ of $A_{n}$ given by (5.1) exists if, and only if, $\cos \phi=-b / 2 \rho c$ for some $\phi \in \mathbb{C}$ and $\rho^{n} \sin (n+1) \phi-\sin \phi \neq 0$. Furthermore, if it exists, then

$$
\begin{aligned}
g_{j}^{(k)}= & \frac{-\rho^{j-k}}{\rho c \sin \phi\left(\sin (n+1) \phi-\rho^{-n} \sin \phi\right)} \\
& \times \begin{cases}\sin j \phi \sin (n+1-k) \phi, & j<k, \\
\sin k \phi \sin (n+1-j) \phi+\rho^{-n} \sin (j-k) \phi \sin \phi, & j \geq k .\end{cases}
\end{aligned}
$$

(ii) Suppose $b^{2}=4 a c$. Then the inverse $G_{n}$ of $A_{n}$ given by (5.1) exists if, and only if, $\rho^{n}(n+1)-1 \neq 0$ and, if it exists,

$$
g_{j}^{(k)}=\frac{-\rho^{j-k}}{\rho c\left(n+1-\rho^{-n}\right)} \times \begin{cases}j(n+1-k), & j<k, \\ k(n+1-j)+\rho^{-n}(j-k), & j \geq k\end{cases}
$$


Suppose $\alpha=0$ and $\beta=-c$ in the matrix $A_{n}$.

(i) Suppose $b^{2} \neq 4 a c$. Then the inverse $G_{n}$ of $A_{n}$ given by (5.1) exists if, and only if, $\cos \phi=-b / 2 \rho c$ for some $\phi \in \mathbb{C}$ and $\rho^{n} \sin (n+1) \phi+\sin \phi \neq 0$. Furthermore, if it exists, then

$$
\begin{aligned}
g_{j}^{(k)}= & \frac{-\rho^{j-k}}{\rho c \sin \phi\left(\sin (n+1) \phi+\rho^{-n} \sin \phi\right)} \\
& \times \begin{cases}\sin j \phi \sin (n+1-k) \phi, & j<k, \\
\sin k \phi \sin (n+1-j) \phi-\rho^{-n} \sin (j-k) \phi \sin \phi, & j \geq k .\end{cases}
\end{aligned}
$$

(ii) Suppose $b^{2}=4 a c$. Then the inverse $G_{n}$ of $A_{n}$ given by (5.1) exists if, and only if, $\rho^{n}(n+1)+1 \neq 0$ and, if it exists,

$$
g_{j}^{(k)}=\frac{-\rho^{j-k}}{\rho c\left(n+1+\rho^{-n}\right)} \times \begin{cases}j(n+1-k), & j<k, \\ k(n+1-j)-\rho^{-n}(j-k), & j \geq k .\end{cases}
$$

\subsection{The case where $\alpha= \pm a, \beta= \pm c$}

THEOREM 6.3. Suppose $\alpha=a$ and $\beta=c$ in the matrix $A_{n}$.

(i) Suppose $b^{2} \neq 4 a c$. Then the inverse $G_{n}$ of $A_{n}$ given by (5.1) exists if, and only if, $\cos \phi=-b / 2 \rho c$ for some $\phi \in \mathbb{C}$ and $\rho^{2 n}-2 \rho^{n} \cos n \phi+1 \neq 0$. Furthermore, if it exists, then

$$
\begin{aligned}
g_{j}^{(k)}= & \frac{\rho^{j-k}}{\rho c \sin \phi\left(\rho^{n}-2 \cos n \phi+\rho^{-n}\right)} \\
& \times \begin{cases}\rho^{n} \sin (k-j) \phi+\sin (n+j-k) \phi, & j<k, \\
\rho^{-n} \sin (j-k) \phi+\sin (n+k-j) \phi, & j \geq k .\end{cases}
\end{aligned}
$$

In particular, when $a=c$,

$$
g_{j}^{(k)}=\frac{\sin (|k-j| \phi)+\sin (n-|k-j|) \phi}{2 a \sin \phi(1-\cos n \phi)} .
$$

(ii) If $b^{2}=4 a c$ and $\rho^{n} \neq 1$, then the inverse $G_{n}$ given by (5.1) exists and

$$
g_{j}^{(k)}=\frac{\rho^{j-k}}{\rho c\left(\rho^{n}-2+\rho^{-n}\right)} \times \begin{cases}\rho^{n}(k-j)+(n+j-k), & j<k, \\ \rho^{-n}(j-k)+(n+k-j), & j \geq k .\end{cases}
$$

In particular, when $b=2 a=2 c$ and $n$ is odd, then $g_{j}^{(k)}=(-1)^{j-k}(n-2|k-j|) / 4 a$. If $b=2 a=2 c$ and $n$ is even, or $b=-2 a=-2 c$, then $\rho^{n}=1$ and the matrix $A_{n}$ is singular. 
Suppose $\alpha=-a$ and $\beta=-c$ in the matrix $A_{n}$.

(i) Suppose $b^{2} \neq 4 a c$. Then the inverse $G_{n}$ of $A_{n}$ given by (5.1) exists if, and only if, $\cos \phi=-b / 2 \rho c$ for some $\phi \in \mathbb{C}$ and $\rho^{2 n}+2 \rho^{n} \cos n \phi+1 \neq 0$. Furthermore, if it exists, then

$$
\begin{aligned}
g_{j}^{(k)}= & \frac{\rho^{j-k}}{\rho c \sin \phi\left(\rho^{n}+2 \cos n \phi+\rho^{-n}\right)} \\
& \times \begin{cases}\rho^{n} \sin (k-j) \phi-\sin (n+j-k) \phi, & j<k, \\
\rho^{-n} \sin (j-k) \phi-\sin (n+k-j) \phi, & j \geq k\end{cases}
\end{aligned}
$$

In particular, when $a=c$,

$$
g_{j}^{(k)}=\frac{\sin (|k-j| \phi)-\sin (n-|k-j|) \phi}{2 a \sin \phi(1+\cos n \phi)} .
$$

(ii) If $b^{2}=4 a c$ and $\rho^{n} \neq-1$, then the inverse $G_{n}$ of $A_{n}$ exists and

$$
g_{j}^{(k)}=\frac{\rho^{j-k}}{\rho c\left(\rho^{n}+2+\rho^{-n}\right)} \times \begin{cases}\rho^{n}(k-j)-(n+j-k), & j<k, \\ \rho^{-n}(j-k)-(n+k-j), & j \geq k .\end{cases}
$$

In particular, if $b=-2 a=-2 c$, then

$$
g_{j}^{(k)}=(2|k-j|-n) / 4 a .
$$

If $b=2 a=2 c$ and $n$ is even, then

$$
g_{j}^{(k)}=(-1)^{j-k}(n-2|k-j|) / 4 a .
$$

If $b=2 a=2 c$ and $n$ is odd, then $\rho^{n}=-1$ and the matrix $A_{n}$ is singular.

\section{Examples}

We give two applications of our explicit formulas.

EXAMPLE 1. In the synchronisation problems of artificial neural networks [11], we encounter the tridiagonal Toeplitz matrix with $a=c=1, b=-2$ with the corners $\alpha=\beta=-1$. Theorem 4.3 gives the eigenvalues and eigenvectors for such matrices. As a numerical example, consider the following matrix:

$$
A_{4}=\left(\begin{array}{cccc}
-2 & 1 & 0 & -1 \\
1 & -2 & 1 & 0 \\
0 & 1 & -2 & 1 \\
-1 & 0 & 1 & -2
\end{array}\right)
$$


By Theorem 4.3, we have

$$
\lambda_{k}=-2+2 \cos \frac{(2 k-1) \pi}{4}, \quad k=1,2,3,4,
$$

which gives $\lambda_{1}=\lambda_{4}=-2+\sqrt{2}$ and $\lambda_{2}=\lambda_{3}=-2-\sqrt{2}$. Since $a=c$, the eigenvectors may be obtained by either (4.17) or (4.18). By (4.17), we have

$$
u^{(1)}=\left(\frac{\sqrt{2}}{2}-i \frac{\sqrt{2}}{2},-i,-\frac{\sqrt{2}}{2}-i \frac{\sqrt{2}}{2},-1\right)^{\dagger} .
$$

By (4.18), if we take $c_{1}=1$ and $c_{2}=0$, then $u^{(1)}=(\sqrt{2} / 2,0,-\sqrt{2} / 2,-1)^{\dagger}$; if we take $c_{1}=0$ and $c_{2}=1$, then $u^{(1)}=(\sqrt{2} / 2,1, \sqrt{2} / 2,0)^{\dagger}$. It can be easily checked that they are the correct eigenvectors corresponding to $\lambda_{1}$.

We remark that our theorem is also applicable when $b=2$. An inspection of (4.16) to (4.18) reveals that only $\lambda_{k}$ depends on $b$. Thus the eigenvalues of the matrix

$$
B_{4}=\left(\begin{array}{cccc}
2 & 1 & 0 & -1 \\
1 & 2 & 1 & 0 \\
0 & 1 & 2 & 1 \\
-1 & 0 & 1 & 2
\end{array}\right)
$$

are given by $\lambda_{1}=\lambda_{4}=2+\sqrt{2}$ and $\lambda_{2}=\lambda_{3}=2-\sqrt{2}$, with the corresponding eigenvectors unchanged.

The inverses of (7.1) and (7.2) may be obtained by (6.4) and (6.5) in Theorem 6.3 as

$$
A_{4}^{-1}=\frac{1}{2}\left(\begin{array}{cccc}
-2 & -1 & 0 & 1 \\
-1 & -2 & -1 & 0 \\
0 & -1 & -2 & -1 \\
1 & 0 & -1 & -2
\end{array}\right) \quad \text { and } \quad B_{4}^{-1}=\frac{1}{2}\left(\begin{array}{cccc}
2 & -1 & 0 & 1 \\
-1 & 2 & -1 & 0 \\
0 & -1 & 2 & -1 \\
1 & 0 & -1 & 2
\end{array}\right)
$$

respectively.

EXAMPLE 2. Consider the following three-point boundary value problem [12] of the form

$$
\begin{cases}\Delta^{2} u_{k-1}+u_{k}=f\left(u_{k}\right), & k=1,2, \ldots, n, \\ u_{0}=\alpha u_{l}, & u_{n+1}=0 .\end{cases}
$$

In matrix form, this may be written as

$$
A_{n} u=F(u)
$$


where $u=\left(u_{1}, \ldots, u_{n}\right)^{\dagger}, F(u)=\left(f\left(u_{1}\right), \ldots, f\left(u_{n}\right)\right)^{\dagger}$ and

$$
A_{n}=\left(\begin{array}{cccccccc}
-1 & 1 & 0 & \cdots & \alpha & \cdots & 0 & 0 \\
1 & -1 & 1 & \cdots & 0 & \cdots & 0 & 0 \\
0 & 1 & -1 & \cdots & 0 & \cdots & 0 & 0 \\
\vdots & \vdots & \vdots & \vdots & \vdots & \vdots & \vdots & \vdots \\
0 & 0 & 0 & \cdots & 0 & \cdots & -1 & 1 \\
0 & 0 & 0 & \cdots & 0 & \cdots & 1 & -1
\end{array}\right)_{n \times n}
$$

A necessary condition for the system (7.3) to be solvable is that $A_{n}$ is invertible. In the case where $l=n$ and $\alpha= \pm 1$, Theorem 6.1 offers some help. As a numerical example for $l=n=6$ and $\alpha=-1$, since $\rho=1$,

$$
\phi=\cos ^{-1} \frac{1}{2}=\frac{\pi}{3} \quad \text { and } \quad\left(\sin \frac{\pi}{3}\right)\left(\sin \frac{7 \pi}{3}+\sin \frac{\pi}{3}\right)=\frac{3}{2} \neq 0,
$$

we see that the inverse exists and is given by (6.3) as

$$
g_{j}^{(k)}=\frac{-2}{3} \times \begin{cases}\sin \frac{j \pi}{3} \sin \frac{(7-k) \pi}{3}-\sin \frac{(k-j) \pi}{3} \sin \frac{\pi}{3} & j<k, \\ \sin \frac{k \pi}{3} \sin \frac{(7-j) \pi}{3} & j \geq k\end{cases}
$$

which yields

$$
\left(\begin{array}{cccccc}
-1 & 1 & 0 & 0 & 0 & -1 \\
1 & -1 & 1 & 0 & 0 & 0 \\
0 & 1 & -1 & 1 & 0 & 0 \\
0 & 0 & 1 & -1 & 1 & 0 \\
0 & 0 & 0 & 1 & -1 & 1 \\
0 & 0 & 0 & 0 & 1 & -1
\end{array}\right)=\frac{1}{2}\left(\begin{array}{cccccc}
0 & 2 & 2 & 0 & -2 & -2 \\
1 & 1 & 2 & 1 & -1 & -2 \\
1 & 1 & 0 & 1 & 1 & 0 \\
0 & 0 & 0 & 0 & 2 & 2 \\
-1 & -1 & 0 & 1 & 1 & 2 \\
-1 & -1 & 0 & 1 & 1 & 0
\end{array}\right) .
$$

\section{References}

[1] S. S. Cheng, Partial Difference Equations (Taylor and Francis, London, 2003).

[2] S. S. Cheng and L. Y. Hsieh, "Inverses of matrices arising from difference operators", Util. Math. 38 (1990) 65-77.

[3] P. J. Davis, Circulant Matrices (Wiley and Sons, New York, 1979).

[4] M. Dow, "Explicit inverses of Toeplitz and associated matrices", ANZIAM J. 44 (2003) 185-215.

[5] I. C. Gohberg and A. A. Semencul, "On the inversion of finite Toeplitz matrices and their continuous analogs”, Mat. Issled. 7 (1972) 201-223, (in Russian). 
[6] R. T. Gregory and D. Karney, A collection of matrices for testing computational algorithms (WileyInterscience, New York, 1969).

[7] S. B. Haley, "Solutions of band matrix equations by projection-recurrence", Linear Algebra Appl. 32 (1980) 33-48.

[8] A. S. Householder, The Theory of Matrices in Numerical Analysis (Dover Publications, New York, 1975).

[9] W. F. Trench, "An algorithm for the inversion of finite Toeplitz matrices", SIAM J. Appl. Math. 12 (1964) 515-522.

[10] W. C. Yueh, "Eigenvalues of several tridiagonal matrices", Appl. Math. E-Notes 5 (2005) 66-74.

[11] W. C. Yueh and S. S. Cheng, "Synchronization in an artificial neural network", Chaos Solitons Fractals preprint.

[12] G. Zhang and R. Medina, "Three-point boundary value problems for difference equations", Comput. Math. Appl. 48 (2004) 1791-1799. 